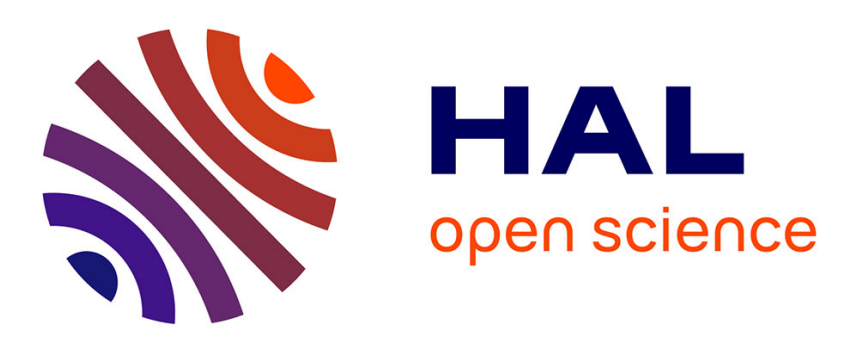

\title{
Visible Light Communications Cooperative Architecture for the Intelligent Transportation System
}

Alin Cailean, Barthélemy Cagneau, Luc Chassagne, Suat Topsu, Yasser

Alayli, Mihai Dimian

\section{- To cite this version:}

Alin Cailean, Barthélemy Cagneau, Luc Chassagne, Suat Topsu, Yasser Alayli, et al.. Visible Light Communications Cooperative Architecture for the Intelligent Transportation System. 20th IEEE Symposium on Communications and Vehicular Technology, SCVT, 2013, Namur, Belgium. pp.1-5. hal-01207174

\section{HAL Id: hal-01207174 \\ https://hal.science/hal-01207174}

Submitted on 30 Sep 2015

HAL is a multi-disciplinary open access archive for the deposit and dissemination of scientific research documents, whether they are published or not. The documents may come from teaching and research institutions in France or abroad, or from public or private research centers.
L'archive ouverte pluridisciplinaire HAL, est destinée au dépôt et à la diffusion de documents scientifiques de niveau recherche, publiés ou non, émanant des établissements d'enseignement et de recherche français ou étrangers, des laboratoires publics ou privés. 


\section{Visible Light Communications Cooperative Architecture for the Intelligent Transportation System}

\author{
Alin-M. Cailean ${ }^{1,2}$, Barthélemy Cagneau ${ }^{1}$, Luc \\ Chassagne $^{1}$, Suat Topsu ${ }^{1}$, Yasser Alayli ${ }^{1}$ \\ ${ }^{1} \mathrm{LISV}$ \\ University of Versailles Saint-Quentin \\ Vélizy, France \\ \{barthelemy.cagneau, luc.chassagne\}@uvsq.fr
}

\author{
Mihai Dimian ${ }^{2}$ \\ ${ }^{2}$ Department of Computers, Electronics and Automation \\ Stefan cel Mare University \\ Suceava, Romania
}

\begin{abstract}
This paper presents the experimental results for a cooperative system that uses Infrastructure-to-Vehicle (I2V) and Vehicle-to-Vehicle (V2V) communication to increases the traffic safety, efficiency and adaptability. The wireless communication technique chosen to enable the data transmission is Visible Light Communication (VLC) due to its numerous advantages. The system consists of a commercial LED-based traffic light as a Road-Side-Unit (RSU) emitter, a transceiver that receives the message from the traffic light and retransmits it to the vehicle behind by using the tail lights, and also a second receiver that receives the message from the transceiver. Measurements exhibit a Bit Error Ratio of $\mathbf{1 0}^{-7}$, both for the I2V and V2V, without using any complex error correction codes, which is considered good, even for traffic safety applications. The tests results show that the proposed cooperative system allows the RSU to communicate with vehicles outside the service area by using multi-hop communication.
\end{abstract}

Keywords - LED; Intelligent Transportation System; Visible Light Communications; cooperative system; VANET.

\section{INTRODUCTION}

The number of vehicles that use the transportation infrastructure increases with every year. Because of this reason there is the mandatory need to continuously improve the safety and the efficiency of the transportation system.

Even if the auto industry has progressed a lot, and today's cars are safer than ever before, road accidents kill more people with every year. More than 1.2 million people die every year because of car accidents, while 20 to 50 million are injured. Road accidents are the leading cause of death among young people aged between 15 to 29 years. Furthermore, the forecasts are even worse: it is estimated that by 2020 road accidents will be the sixth cause of death with 1.9 million victims yearly $[1,2]$. This clearly demonstrates that besides the individual safety mechanisms, there is a strong requirement for a complex cooperative system in which all the components contribute to increase not just their own safety but the safety of the entire transportation system.

Intelligent Transportation System (ITS) integrates state-ofthe-art cooperative technologies to increase the safety and efficiency of the transportation system and also to reduce the $\mathrm{CO}_{2}$ emissions. By enabling wireless communications among vehicles and between vehicles and infrastructure the safety and

This work was supported in part by the University of Versailles SaintQuentin and Valeo Industry.

A part of the financial support is granted by the Fond Unique Interminsteriel (FUI) project named Co-Drive, supported by the Pôle de Compétitivité Mov’eo. the efficiency of road traffic can be substantially improved. Inter-Vehicle Communication (IVC) or Vehicle-to-Vehicle Communication (V2V) systems allow modern vehicles to communicate with each other and to share information regarding their mechanical state (e.g. position, velocity, acceleration, engine state, etc) or information about the traffic (e.g. state of traffic-lights, accidents, traffic jams, line works, etc). At the same time IVC systems have the potential to improve the passenger's comfort. Infrastructure-to-Vehicle (I2V) communications allow the cooperation between road infrastructure and vehicles. Besides these communication technologies, ITS integrates a Cooperative Traffic Control and Management Center that gathers the data from the traffic, analyses it, takes the required corrective measures and redistributes the information. In this Vehicle Ad-hoc Network (VANET) [3], the vehicles represent the mobile nodes whereas the RSU, as part of the traffic infrastructure, represent the fixed gateways. In order the system to be able to work at maximum potential it needs a high degree of market penetration and a large geographical distribution.

ITS involves cooperative driving technologies, based on wireless communication that allow vehicles and/or road infrastructure to exchange a large amount of dynamic which will generate a large data flow. A serious problem is represented by the fact that the wireless communication technologies, on which the cooperative driving relies on, are known to be subject for different type of interferences. This problem is even more acute in the case of VANETs, where different nodes will cause mutual interferences. Under these circumstances, the Line-of-Sight (LoS) condition which is a major disadvantage of Visible Light Communication (VLC), limiting the communication range, may act here as an advantage, preventing interferences or the phenomena called "broadcasting storm"[4]. In these conditions, the major challenge for the ITS, is to be reliable and ubiquitous but at the same time to keep the implementation cost as low as possible.

The main novelty of this paper consists in the experimental demonstration of the cooperation between two major components of the ITS: I2V and V2V communications. We consider that the proposed architecture has the advantage of limiting the interferences caused by multiple emitters that are close to each other, and also has the potential to increase the 
communication range by using node to node communication. The proposed system achieves a promising Bit Error Ratio (BER) of $10^{-7}$ for the both I2V and V2V communication which could be improved if required, by integrating error detection and correction codes and protocols. Another advantage of the proposed system is its ability to maintain the low BER while increasing the emitter-receiver distance.

\section{THE POTENTIAL ROLE OF VLC IN THE ITS}

In order to enable V2V and V2I communications several technologies have been proposed and explored: 802.11 (WiFi) [5], Bluetooth [6], VLC [7]. V2V communications based on RF are very likely to present severe collisions in high traffic density, when multiple vehicles use the same medium $[8,9]$, and this leads to unaccepted latencies for a reliable safety application, which require latencies less than $100 \mathrm{~ms}$ [10]. Furthermore, the radiofrequency channels are often saturated and the extension of new available carriers is expensive or even impossible. However, in low density traffic, $\mathrm{RF}$ based solutions have the advantage of being able to communicate at greater distances than VLC solutions.

VLC is provided by LED lighting systems. The LEDs outperform the classical lighting sources in terms of energy efficiency, reliability and life-time. Due to the numerous advantages offered by the LEDs, the automotive industry had begun to replace the halogen lamps by LED lighting systems. The LED lighting also began being used for LED-based traffic lights. These new traffic lights come with a low maintenance cost, long life, low energy consumption and also have better visibility. Considering the trends in the lighting industry, it is expected that in the near future, the street lighting will be LEDs based, so the road illumination will also be able to provide with communication possibilities.

In the light of the upper mentioned, one can see that LEDbased lighting will be part of the transportation system, being integrated into vehicles and also in the infrastructure. The large geographical area in which LEDs lighting will be used, combined with the VLC technology will allow the ITS to gather data from a widespread area and will enable the distribution of high quality information. These additional functions are possible without affecting in any way the primary goal, which is signaling or lighting.

The success of the ITS is largely dependent on its penetration. Insufficient penetration means insufficient data collection and distribution. If it is to think of RF solutions for the ITS, this will not be possible for a long time ahead because, in order the system to be effective, it is required that all the intersections and the streets to be equipped with RF units, which implies a huge implementation cost. VLC has the advantage that the LEDs traffic lights and the street lighting will already be at every intersection.

Besides the ubiquitous nature, VLC has other advantages. VLC uses the visible light spectrum as a communication medium and since there are no regulations that limit the use of the light spectrum, VLC has the potential to reach extremely high data rates [11] thanks to the huge available bandwidth. Another advantage is that whereas radio waves are considered harmful to the human body and infra-red light is dangerous for the human eye, VLC is safe for the human health.

In order to extract the data from the light beam, the VLC receiver uses a light sensing element, which can be either a camera system, or a photodetector element. Camera systems are used combined with complex image or pixel processing applications as in [12]. Even though such systems are able to achieve good performances for BER over tens of meters, using a high-speed camera raises the implementation costs limiting these types of solutions, mostly for laboratory prototypes.

Concerning the work regarding the VLC between infrastructures and vehicles, this was mainly focused on communication between traffic light and vehicle [13], mainly because the high power of traffic light, which allows for long distance transmissions. For the receiving part, the noise performances of photosensing elements like photodetectors allow them to be used even for long ranges. An optical part is most of the times used for focusing the light. Active control of the position of the sensing element has been achieved to enhance the BER [14]. At shorter ranges, photosensing elements are efficient and do not require active control of the position.

\section{EXPERIMENTAL CONFIGURATION}

The LISV (Laboratoire d'Ingénierie des Systèmes de Versailles) of the University of Versailles is involved in a French consortium working on automated and instrumented vehicles. A patent has been deposited on optoelectronics system for communication and inter-vehicle distance measurements [15].

For the purpose of this experiment, two prototypes of ledlight communications have been implemented. The aim was to enable the cooperation between the two communication systems. The first one is an example of I2V communication, between a commercial LED traffic light as a RSU emitter and a transceiver. The second one is an example of $\mathrm{V} 2 \mathrm{~V}$ communication and uses a vehicle's rear-light emitter, to transmit to the vehicle behind, the original message, received from the traffic light, with the mention that additional information can be added. Both the prototypes transmit the digital information by using power modulation, which is the most appropriate for wireless optical links. Even if by this time, the electronics are not embedded, all the components have been chosen for their low cost, their compactness and are compatible with industrial prototypes.

The proposed cooperative system has several advantages. First, it enables short to medium communication between road infrastructure and also among vehicles without causing mutual interferences. The message is forwarded from node to node, so it can reach to network nodes (vehicles) that are outside the communication area. So, by using multi-hop networking both LoS problem and limited communication range are solved. This scenario is presented in Fig. 1, where the first vehicle, which is in the Service Area (SA) of the traffic light, retransmits the received message to the vehicle behind, which is outside the traffic light's SA. 


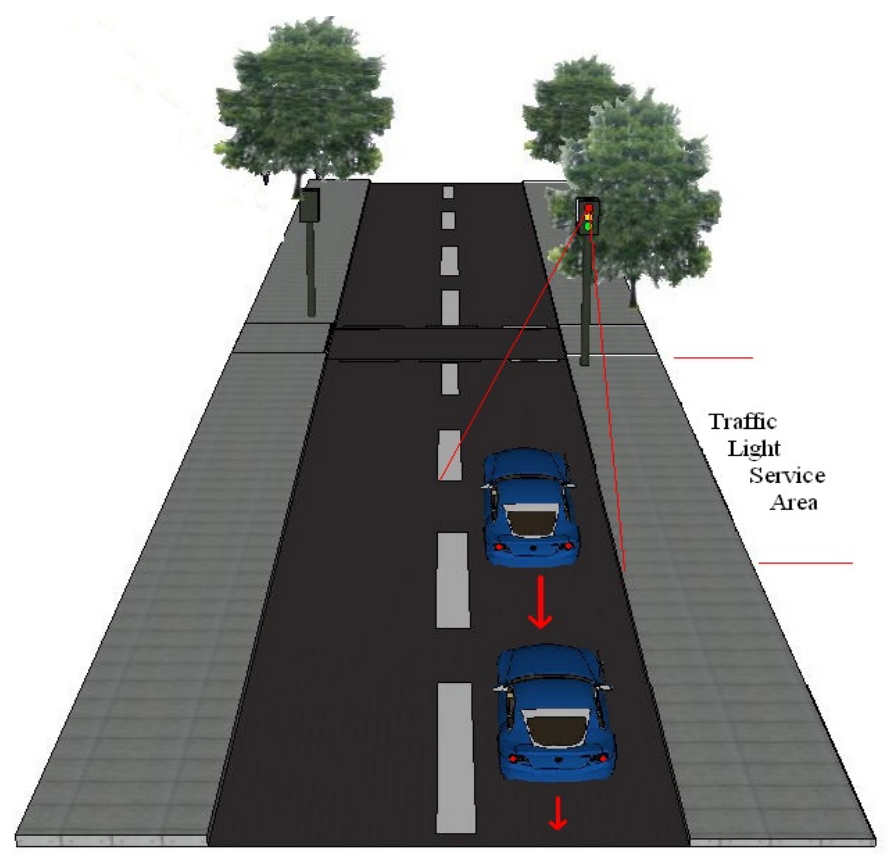

Figure 1: Illustration of the proposed scenario: the traffic light sends a message that is received by the first car and retransmitted to the car behind.

\section{A. Considerations on the coding}

Regarding the coding, we chose to implement two codes: the biphase (Manchester code) and the Miller code. Both the codes use OOK (On-Off Keying) amplitude modulation which is simple and well suited for data transmissions at frequencies of tens of kilohertz. The prototype allows switching between Manchester and Miller code, so different types of tests can be performed.

The Manchester code is a classical one, and is recommended for outdoor optical communications [16]. The Miller code is easily constructed using the biphase code. Even though Miller code is not as popular as Manchester, we decided to use it since it appears to be convenient for MIMO applications. As showed in [17], having the Power Spectral Density (PSD) centered in a short bandwidth range, makes the Miller code appropriate for multiple channels communications.

\section{B. Considerations on the I2V communication system}

The RSU emitter consists of a commercial LED-based traffic light on which we made slight adjustments. We chose this approach in order to demonstrate how easily a standard traffic light can become a data broadcast station. For VLC, any source of light can become an information broadcast unit without affecting the original purpose of signaling. We have added a low-cost microcontroller (Microchip PIC18F2550) that deals with the data encoding and packaging. The microcontroller controls a digital power switch that handles the switching of the LEDs. The modulation frequency for these experiments is $15 \mathrm{kHz}$ but it can be increased if required.

The structure of the frame is a classical one, as it can be seen in Fig. 2. The message begins with several synchronization bits to inform the receiving board that a message has been sent. The rest of the frame consists of start and stop bits, an additional flag that contains the length of the frame and of course, the data bytes.

Normally, the traffic light is continuously transmitting the current color and also the remaining time until the next color change. This information helps increasing the traffic safety and also is useful for engine regulation adjustment on intelligent vehicles. To facilitate the communication with the emitter, the microcontroller is interfaced with a PC through USB in the same manner that a RSU has to be connected with the Traffic Control and Management Center. This way, new data can be broadcasted easily at any moment (e.g. alternative routes, traffic jams, etc).

The representation of the $\mathrm{I} 2 \mathrm{~V}$ communication is shown in Fig.2.

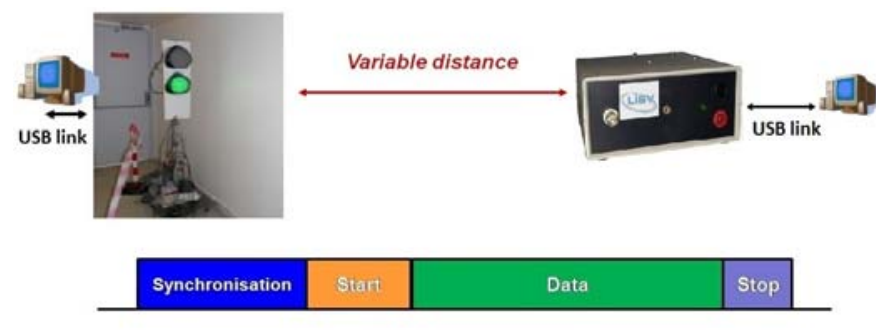

Figure 2: Working principle for I2V prototype.

\section{Considerations on the V2V communication system}

The C2C communication is represented in Fig. 3 and basically works on the same principle as the I2V communication. The data transmitted by the RSU is received by a transceiver that extracts the data from the light beam and also retransmits it to vehicles that are behind it, by using the rear lights. The transceiver basically consists of a receiver module and an emitter module. The power emitted by the tail light is weaker compared with the power of the traffic light, which limits the communication range for the V2V prototype. The range could be increased, but still it will be shorter than the I2V communication range.
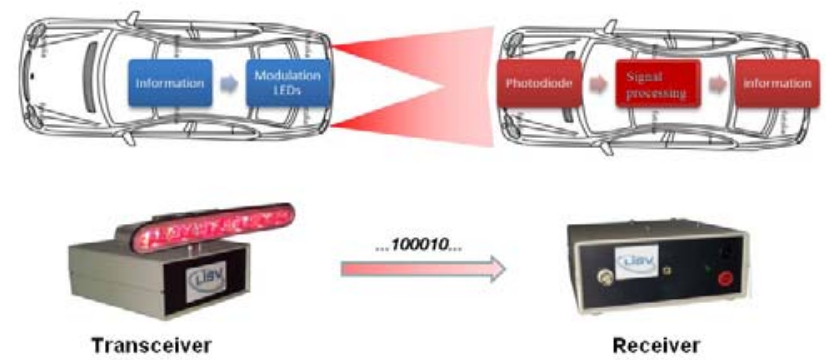

Figure 3: Working principle for V2V prototype.

\section{Considerations on the reception module}

The receiver module (Fig. 4) uses a low cost silicon photodetector as a light sensing element. To condition the sensitive element, we use a classical transimpedance circuit. The photodiode is coupled with low cost pre-amplifier electronics. 


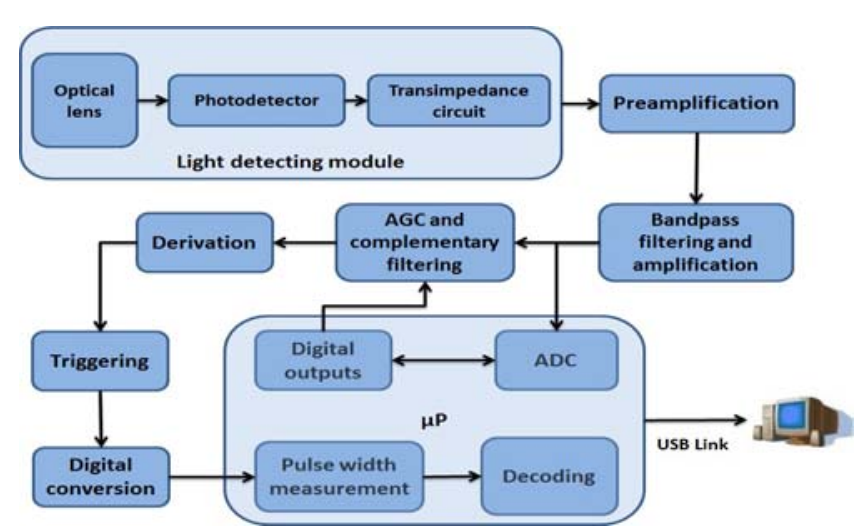

Figure 4: Synopsis of the VLC receiver.

The receiving system aims to be able to extract data at short to medium distances whatever the environmental conditions. Once the signal to noise is ensured, the signal is pre-amplified to ensure minimum magnitude levels around tens millivolts whatever the distance. An analog band-pass filter suppresses the offset due to the daylight and filters high frequencies noise. Then, the ADC (Analog-to-Digital Converter) included in a low cost microcontroller (microchip Pic family) digitalizes the signal. To be able to properly receive data at both short and long distances, the receiver integrates an Automatic Gain Control (AGC) stage responsible for gain selection. Depending on the level of the signal, the AGC processes the gain, so that photodiode saturation is prevented at short distance whereas insufficient amplification is prevented at long distance. Besides the complementary gain, this stage comes with complementary filtering.

When the signal reaches the required amplitude, it is derived with a high-pass filter. The derivative of a square signal leads to alternated positive and negative pulses. Based on these pulses, the signal is triggered and the system converts the signal into two-level digital signals (light on and light off). It is then sent to the microcontroller to measure the width of the pulses with the precise clock of an external $20 \mathrm{MHz}$ external quartz crystal. The decoding is made in real time, based on the pulse length. The message is then retransmitted to the vehicle behind by using the tail lights. Additional data may be added if needed (e.g. vehicle identification, GPS coordinates, etc.).

\section{EXPERIMENTAL RESULTS}

The experiments were conducted in laboratory conditions. The traffic light transmits data sets of 10 million bits since it can be considered that a BER lower than $10^{-7}$ is good even in road safety applications. The message transmitted contains 7 ASCII characters of 8 bits, however longer messages can be send. The frame of the message indicates to the receivers if Miller or Manchester code is used. The transceiver receives the data and decodes it in real-time. The transceiver also resends the message for the second receiver by using the tail lights. An algorithm allows post-processing or calculation of errors to determine the BER. The BER is determined by comparing the received bits with the emitted ones.

For these experiments a predefined message is sent continuously at a $15 \mathrm{kHz}$ modulation frequency. The synchronization between the emitter and receiver is not required since the clock of the microcontroller is an accurate and stable one. For simplicity and low cost, we chose to use an asynchronous link which does not require the transmission of the clock.

Two scenarios were tested. In the first scenario, the transceiver is situated in the traffic light's SA whereas the second receiver is situated outside the traffic light's SA. We began the experiments by setting the transceiver 20 meters away from the traffic light and the receiver 1 meter behind it, with no LoS with the traffic light. We then gradually increased the distances between the transceiver and receiver and measured the BER. Due to space limitation imposed by the building, the distances involved were limited, but the purpose of the experiment was to demonstrate that VLC communication can reach to a vehicle outside the service area. The results obtained for this scenario are presented in Table I.

Table I: Bit Error Ratio (BER) for Miller and Manchester codes at $15 \mathrm{kHz}$ for Scenario 1

\begin{tabular}{|c|c|c|c|}
\hline Communication & $\begin{array}{c}\text { Distance } \\
{[\mathrm{m}]}\end{array}$ & $\begin{array}{c}\text { BER for } \\
\text { Manchester }\end{array}$ & $\begin{array}{c}\text { BER for } \\
\text { Miller }\end{array}$ \\
\hline $\mathrm{I} 2 \mathrm{~V}$ & 20 & \multirow{7}{*}{$<10^{-7}$} & \multirow{7}{*}{$<10^{-7}$} \\
\hline \multirow{3}{*}{$\mathrm{V} 2 \mathrm{~V}$} & 1 & & \\
\hline & 2 & & \\
\hline & 3 & & \\
\hline \multirow{3}{*}{$\mathrm{I} 2 \mathrm{~V} 2 \mathrm{~V}$} & 21 & & \\
\hline & 22 & & \\
\hline & 23 & & \\
\hline
\end{tabular}

In the second scenario, both the transceiver and the receiver are situated in the traffic light's SA, but there is no LoS between the traffic light and the receiver. We set the transceiver 1 meter away from the traffic light and the receiver 1 meter behind it. In the next steps, we increased the distance between transceiver and receiver and also varied the distance between the traffic light and transceiver. The BER was processed both for the I2V and for V2V communication and the results are presented in Table II.

Table II: Bit Error Ratio (BER) for Miller and Manchester codes at $15 \mathrm{kHz}$ modulation frequencies for $\mathrm{I} 2 \mathrm{~V}, \mathrm{~V} 2 \mathrm{~V}$, and $\mathrm{I} 2 \mathrm{~V} 2 \mathrm{~V}$

\begin{tabular}{|c|c|c|c|c|}
\hline $\begin{array}{c}\text { Distance } \\
\text { I2V } \\
\text { [m] }\end{array}$ & $\begin{array}{c}\text { Distance } \\
\text { V2V } \\
\text { [m] }\end{array}$ & $\begin{array}{c}\text { Distance } \\
\text { I2V2V } \\
(\mathrm{I} 2 \mathrm{~V}+\mathrm{V} 2 \mathrm{~V}) \\
{[\mathrm{m}]} \\
\end{array}$ & $\begin{array}{c}\text { BER for } \\
\text { Manchester }\end{array}$ & $\begin{array}{c}\text { BER } \\
\text { for } \\
\text { Miller }\end{array}$ \\
\hline \multirow{3}{*}{1} & 1 & 2 & \multirow{12}{*}{$<10^{-7}$} & \multirow{12}{*}{$<10^{-7}$} \\
\hline & 2 & 3 & & \\
\hline & 3 & 4 & & \\
\hline \multirow{3}{*}{5} & 1 & 5 & & \\
\hline & 2 & 7 & & \\
\hline & 3 & 8 & & \\
\hline \multirow{3}{*}{10} & 1 & 11 & & \\
\hline & 2 & 12 & & \\
\hline & 3 & 13 & & \\
\hline \multirow{3}{*}{15} & 1 & 16 & & \\
\hline & 2 & 17 & & \\
\hline & 3 & 18 & & \\
\hline
\end{tabular}

To test the immunity to parasitic signals, tests were performed inside a corridor with artificial lights on. The neon 
lights provide a strong $100 \mathrm{~Hz}$ parasitic signal which is successfully eliminated by the filters without having any influence on the $10^{-7} \mathrm{BER}$.

The experimental results show a BER of $10^{-7}$ for both I2V and $\mathrm{V} 2 \mathrm{~V}$ communication for variable distances. These communication distances can be increased especially in the case of the $\mathrm{I} 2 \mathrm{~V}$ where the power emitted by the traffic light is high enough to allow longer distances. For such a communication we had obtained a BER of $10^{-7}$ for distances up to 50 meters [18] whereas, for V2V the communication range can be increased in this configuration up to 10 meters [17]. Even so, it is difficult to achieve communication ranges comparable with those of radio communication.

The calculated BER of $10^{-7}$ could also be highly improved considering that it was obtained focusing only on hardware techniques. Errors detecting codes, correlation techniques, or redundancy coding frames or protocols are some possible solutions to achieve this. The results demonstrate that the prototypes are well adapted for data transmission over short or medium distances, for $\mathrm{I} 2 \mathrm{~V}$ and for $\mathrm{V} 2 \mathrm{~V}$, using both Manchester and Miller codes. But, the main objective of the experiment was to test and demonstrate the cooperation between an I2V communication system and a V2V communication system which will be the case for the real traffic scenario.

This paper demonstrated that the limitation represented by the LoS condition and the limited communication range of VLC, can be overcome by using multi-hop networking. We showed that the communication between a RSU and a vehicle that is outside the SA of the RSU is possible with the help of a second vehicle that is found inside the SA and that forwards the message. The same working principle could be applied in the case of radio communication. This will allow the emitters to reduce the emission power, just to allow communication with the closest neighbor, without causing interferences to the other vehicles. Of course, in the real traffic case, more complex routing protocols will be required.

\section{CONCLUSION}

In this paper we present the experimental results obtained by a cooperative system that integrates an I2V and a V2V VLC prototypes. Taking into account the benefits of VLC, it is expected that this type of communication will have an important role in road safety applications. Test results show that the cooperation between the two systems increases the communication range, allowing the RSU to communicate with vehicles outside its SA. This approach can help achieve long range communications by combining short and middle range communication systems. Such a system is highly efficient in high traffic densities where neighboring vehicles can cause interferences to each other if the emitted power is too high. The cooperative system also eliminates the limitations imposed by the LoS condition which is a major drawback of VLC. By using VLC, we achieved BERs that are below $10^{-7}$ without using error correcting codes. The results can be highly improved by integrating error correcting codes but this was not the purpose of this work. As a future objective, we plan to finalize the embedded application for experiments in several road configurations with either static or moving vehicles.

\section{REFERENCES}

[1] World Helth Organization. (July 2013). Fact Sheet 310 - The top 10 causes of death.

[2] World Helth Organization. (March 2013). Fact Sheet 358 Road Traffic Injuries.

[3] S. Yousefi, E. Altman, R. El-Azouzi, and M. Fathy, "Analytical Model for Connectivity in Vehicular Ad Hoc Networks", IEEE Transactions on Vehicular Technology, vol. 57, pp. 3341-3356, 2008.

[4] Tonguz, O.K.; Wisitpongphan, N.; Parikh, J.S.; Fan Bai; Mudalige, P.; Sadekar, V.K., "On the Broadcast Storm Problem in Ad hoc Wireless Networks," Broadband Communications, Networks and Systems, 2006. BROADNETS 2006. 3rd International Conference on , vol., no., pp.1,11, 1-5 Oct. 2006

[5] Jerbi, M.; Marlier, P.; Senouci, S.M., "Experimental Assessment of V2V and I2V Communications," Mobile Adhoc and Sensor Systems, 2007. MASS 2007. IEEE Internatonal Conference on , vol., no., pp.1,6, 8-11 Oct. 2007

[6] Sawant, H.; Jindong Tan; Qingyan Yang; Qizhi Wang, "Using Bluetooth and sensor networks for intelligent transportation systems", Intelligent Transportation Systems, 2004. Proceedings. The 7th International IEEE Conference on , vol., no., pp.767,772, 3-6 Oct. 2004.

[7] N. Kumar, N. Lourenço, D. Terra, L.N. Alves, R.L. Aguiar, "Visible Light Communications in Intelligent Transportation Systems“, IEEE Intelligent Vehicules Symposium 2012, 748-753.

[8] S. Eichler, "Performance Evaluation of the IEEE 802.11p WAVE Communication Standard“, IEEE Vehicular Technology Conference, 2007.

[9] A. Agarwal, T.D.C. Little, "Role of Directional Wireless Communication in Vehicular Networks", IEEE Intelligent Vehicules Symposium 2010, 688-693

[10] U.S. Department of Transportation. Vehicle Safety Communications Project Task 3 Final Report. http://www.ntis.gov/.

[11] Shuailong Zhang; Watson, S.; McKendry, J.J.D.; Massoubre, D.; Cogman, A.; Erdan Gu; Henderson, R.K.; Kelly, A.E.; Dawson, M.D., "1.5 Gbit/s Multi-Channel Visible Light Communications Using CMOSControlled GaN-Based LEDs," Lightwave Technology, Journal of , vol.31, no.8, pp.1211,1216, April15, 2013

[12] T. Nagura, T. Yamazato, M. Katayam, T. Yendo, T. Fujii, H. Okada, "Improved decoding methods of visible lights communication system for ITS using led array and high speed camera", Vehicular technology conference, VTC 2010 IEEE 71t, ISSN: 1550-2252,2010.

[13] N. Kumar, D. Terra, N. Lourenço, L.N . Alves, R.L. Aguiar, "Visible light communication for intelligent transportation in road safety applications", Wireless Communications and mobile computing conference (IWCMC), $20117^{\text {th }}$ international, pp. 1513-1518, doi: 10.1109/IWCMC.2011.5982762, 2011.

[14] S. Okada, T. Yendo, T. Yamazato, T. Fujii, M. Tanimoto, Y. Kimura, "On-vehicle receiver for distant visible light road-to-vehicle communication”, Intelligent Vehicle Symposium, IEEE ISSN: 19310587, 2009.

[15] Y. Alayli, S. Topsu, L. Chassagne, J.M. Blosseville, Patent nº9 58694, Communications par phares, 2009.

[16] IEEE Standard for Local and Metropolitan Area Networks--Part 15.7: Short-Range Wireless Optical Communication Using Visible Light, IEEE Standard, 2011, 1-309.

[17] Alin-Mihai Cailean, Barthélemy Cagneau, Luc Chassagne, Suat Topsu, Yasser Alayli, Jean-Marc Blosseville "Visible light communications: application to cooperation between vehicles and road infrastructures", 2012 IEEE Intelligent Vehicles International Symposium, Alcalá de Henares, Spain, (IV'12), June 3-7 2012.

[18] A. Cailean, B. Cagneau, L. Chassagne, S. Topsu, Y. Alayli, M. Dimian "A robust system for Visible Light Communication“, 2013 IEEE Wireless Vehicle Syposium (WiVeC), in press. 